\title{
SELEÇÃO COMBINADA UNIVARIADA E MULTIVARIADA APLICADA AO MELHORAMENTO GENÉTICO DA SERINGUEIRA ${ }^{1}$
}

\author{
REGINALDO BRITO DA COSTA², MARCOS DEON VILELA RESENDE ${ }^{3}$, ANTONIO JOSÉ DE ARAUJO ${ }^{4}$, \\ PAULO DE SOUZA GONÇALVES ${ }^{5}$ e NELSON BORTOLETTO ${ }^{6}$
}

\begin{abstract}
RESUMO - Este trabalho objetivou estimar os coeficientes de herdabilidade e de predição genética, pelo método multivariado, e comparar os ganhos genéticos obtidos por meio da seleção entre e dentro de progênies, individual e combinada, em seringueira (Hevea brasiliensis (Willd. ex Adr. de Juss.) Müell. Arg.). Vinte e duas progênies de meios-irmãos foram plantadas na Estação Experimental de Votuporanga, SP, no delineamento de blocos ao acaso, com cinco repetições e dez plantas por parcela. Aos três anos de idade foram avaliados os caracteres: número de anéis de vasos laticíferos (NA), produção de borracha seca (PB), espessura de casca (EC) e circunferência do caule (CC). Os resultados demonstraram haver variabilidade significativa entre progênies quanto aos caracteres PB, EC e CC. As estimativas de coeficientes de herdabilidade e de predição genética foram mais elevadas nas progênies, com valores mais expressivos quanto aos coeficientes de predição genética. Ganhos genéticos adicionais foram obtidos pela seleção combinada. Embora os referidos ganhos tenham sido de pequena magnitude, recomenda-se o uso do método, por propiciar maior acurácia e ganho genético. Os valores mais expressivos de acurácia e ganho genético foram estimados pelo método multivariado.
\end{abstract}

Termos para indexação: Hevea brasiliensis, análises combinadas, herdabilidade, ganho genético.

\section{UNIVARIATE AND MULTIVARIATE COMBINED SELECTION APPLIED TO HEVEA BREEDING}

\begin{abstract}
The objective of this paper was to estimate the heritability coefficients by the univariate method and genetic prediction by the multivariate method, as well as to compare the genetic gain obtained by individual and combined selection between and within progenies, in rubber tree (Hevea brasiliensis Willd. ex Adr. de Juss.) Müell. Arg.). Twenty two half-sib progenies were established at the Experimental Station, in Votuporanga, São Paulo State, Brazil, following the randomized block design, with five replications and ten plants per plot. Three years after planting, number of latex vessel rings (NR), yield of dry rubber (YB), bark thickness (BT) and stalk circunference (SC) were evaluated. The results showed significant variability among progenies for YB, BT and SC characters. The heritability coefficients and genetic prediction to progenies level were high, with expressive values for the genetic prediction coefficients. Additional genetic gains were obtained by combined selection. Although the genetic gains had been small, this method is recommended, because it gives a better improvement and genetic gain. The most expressive values of improvement and genetic gain were estimated by multivariate method.
\end{abstract}

Index terms: Hevea brasiliensis, combined analysis, heritability, genetic gain.

${ }^{1}$ Aceito para publicação em 16 de março de 1999.

${ }^{2}$ Eng. Florestal, D.Sc., Av. Tamandaré 6000, Jardim Seminário, Caixa Postal 100, CEP 79117-100 Campo Grande, MS. E-mail: desp@unibosco.br

${ }^{3}$ Eng. Agrôn., D.Sc., Embrapa-Centro Nacional de Pesquisa de Florestas (CNPF), Caixa Postal 319, CEP 83411-000 Colombo, PR.

${ }^{4}$ Eng. Florestal, Ph.D., Universidade Federal do Paraná (UFPR), Dep. de Silvicultura e Manejo, Caixa Postal 886, CEP 80001-970 Curitiba, PR.

${ }^{5}$ Eng. Agrôn., D.Sc., Embrapa-Programa Integrado de São Paulo, Programa Seringueira, Centro de Café e Plantas Tropicais, Instituto Agronômico (IAC), Caixa Postal 28, CEP 13001-970 Campinas, SP. E-mail: paulog@cec.iac.br

${ }^{6}$ Eng. Agrôn., IAC/Núcleo de Agronomia do Noroeste, Caixa Postal 401, CEP 15500-000 Votuporanga, SP.

\section{INTRODUÇÃO}

A seringueira [Hevea brasiliensis (Willd. ex Adr. de Juss.) Muell. Arg.] tem como principais áreas de cultivo, no Brasil, as denominadas áreas de escape do mal-das-folhas e localizam-se nos estados de São Paulo, Mato Grosso do Sul, Mato Grosso, Bahia, e, mais recentemente, no noroeste do Paraná (Paiva \& Kalil Filho, 1985; Pereira, 1992).

Especialmente no Estado de São Paulo a heveicultura experimentou grande expansão na região do planalto, nos municípios de São José do Rio Preto, Barretos, Araçatuba, Bauru, Marília e Presidente Prudente (Virgens Filho, 1990; Sociedade 
Brasileira de Silvicultura, 1995). A seringueira vem se tornando uma cultura de grande importância econômica para o Estado, que é, atualmente, o maior produtor de borracha seca do país (Boock et al., 1995).

O ciclo de melhoramento da seringueira presentemente utilizado compreende diversas etapas de seleção; são necessários cerca de 20 a 25 anos para que se complete o ciclo, desde a obtenção de sementes de polinização aberta ou controlada, até a recomendação de clones para plantio em grande escala (Gonçalves et al., 1990). É reconhecido que o sucesso de um esquema seletivo depende da variação genética disponível na população, e, sobretudo, do valor relativo desta perante a variação não-genética (Paiva, 1992). É de grande interesse a determinação da variabilidade e da herança da produtividade e de caracteres que propiciem ao melhorista a escolha de métodos mais adequados na seleção de plantas jovens, abreviando, assim, o ciclo de melhoramento da espécie.

Os testes de progênies, instrumentos dos mais importantes para o trabalho do melhorista, têm sido usados na estimação dos parâmetros genéticos e seleção de indivíduos entre e dentro de progênies, quando se procura avaliar a magnitude e a natureza da variância genética disponível com vistas a quantificar os ganhos com a seleção, e predizer o melhor método de seleção a ser utilizado.

Nos referidos testes, podem ser discriminados os indivíduos superiores a serem utilizados em novos ciclos de melhoramento. Vencovsky (1987) ressalta que no caso de famílias de meios-irmãos são explorados apenas $25 \%$ da variância aditiva total na seleção entre famílias, ocorrendo, na fase seguinte do programa, a seleção de indivíduos de melhor desempenho dentro de famílias superiores. Segundo Falconer (1987), esta prática proporciona ganhos adicionais por explorar a fração da variância não utilizada na seleção entre, porém apresenta o inconveniente de basear-se em valores fenotípicos individuais, sujeitos a maior influência dos efeitos ambientais.

Os programas de melhoramento genético no Brasil têm-se baseado principalmente na seleção entre e dentro de progênies de meios-irmãos. Nesse esquema, um mesmo número de indivíduos têm sido selecionados em cada uma das famílias, os quais irão compor os pomares de sementes por mudas ou clones (Kageyama, 1980). Esse método de seleção fundamenta-se, portanto, em medidas fenotípicas (médias de famílias e desvio do valor individual).

A seleção combinada, por outro lado, baseia-se em um índice que considera, simultaneamente, o comportamento do indivíduo e sua família. Dessa forma, em função dos pesos de ponderação do indivíduo e da família, é possível selecionar indivíduos superiores, porém de famílias de desempenho intermediário, ou ainda, indivíduos de desempenho intermediário pertencentes a famílias superiores (Falconer, 1987; Resende \& Higa, 1994). A idéia não é nova no melhoramento genético. Lush $(1945,1947)$ realizou a primeira análise dessa questão e propôs a seleção combinada com base em valores individuais com recuperação da informação de famílias. Nesse método, a seleção é baseada em medidas genéticas (valores genéticos líquidos) e não fenotípicas, dos candidatos à seleção.

Nesse contexto, o uso da seleção combinada poderá proporcionar ganhos mais elevados que os obtidos pela seleção massal ou de família. Essa tendência foi demonstrada com relação a outras espécies florestais por Bueno Filho (1992), Resende \& Araujo (1993), Resende \& Bertolucci (1993), Sturion (1993), Sturion et al. (1994), Cornacchia et al. (1995), Pires et al. (1996) e Sampaio (1996). Porém, ressalta-se a inexistência de trabalhos com a seringueira, que comparem seleção entre e dentro de progênies e seleção individual à seleção combinada, visando maximizar o ganho genético.

Este estudo tem como objetivo estimar as magnitudes dos coeficientes de herdabilidade e de predição genética, pelo método multivariado, e comparar os ganhos genéticos obtidos por meio da seleção entre e dentro de progênies, individual e seleção combinada.

\section{MATERIAL E MÉTODOS}

O ensaio foi instalado na Estação Experimental de Votuporanga, SP, pertencente ao Instituto Agronômico de Campinas (IAC), localizada na latitude $20^{\circ} 25^{\prime} \mathrm{S}$, longitude $49^{\circ} 59^{\prime} \mathrm{W}$ e altitude de $450 \mathrm{~m}$. O solo é do tipo Latossolo Vermelho-Escuro, de textura média, em clima tropical continental, com predominância de verão úmido e um período de inverno seco com temperaturas e precipitações pluviais mais reduzidas. A temperatura média anual é de $20^{\circ} \mathrm{C}$ e a pluviosidade média anual é de $1.420 \mathrm{~mm}$. 
O material genético que compõe o estudo é constituído de 22 progênies de meios-irmãos provenientes de sementes de polinização aberta, obtidas de 22 clones parentais selecionados fenotipicamente em uma população-base de $H$. brasiliensis, de origem asiática.

As sementes foram colocadas para germinar em sacos de polietileno, e as progênies foram transportadas para o local definitivo, com dois lançamentos foliares. As progênies foram instaladas sob o delineamento de blocos ao acaso com 22 tratamentos, cinco repetições e dez plantas por parcela, no espaçamento de 1,5 x 1,5 m em linhas simples.

Aos três anos de idade, as progênies foram avaliadas quanto aos caracteres: a) Produção de borracha seca: obtida pelo teste Hamaker-Morris-Mann (HMM), modificado para plântulas de três anos de idade (Tan \& Subramanian, 1976), utilizando-se a média de produção de borracha seca de 30 cortes por plântula. O painel de sangria foi aberto a $20 \mathrm{~cm}$ do solo, pelo sistema $\mathrm{S} / 2 \mathrm{~d} / 3$, num total de 35 cortes, descartando-se as cinco primeiras amostras que correspondem à fase de amansamento do painel. A nomenclatura $\mathrm{S} / 2$ corresponde ao corte em meia espiral, e a nomenclatura $\mathrm{d} / 3$ expressa o intervalo entre sangrias, ou seja, uma sangria a cada três dias; b) Circunferência do caule: determinada a $50 \mathrm{~cm}$ do solo, com o auxílio de fita métrica graduada; as medições foram expressas em centímetro; c) Casca: quanto aos caracteres quantitativos relacionados à casca, foram coletadas duas amostras de casca por planta a $15 \mathrm{~cm}$ de altura do solo, com o auxílio de um vasador. As determinações destes caracteres foram realizadas da seguinte forma: c.1) espessura de casca: obtida com paquímetro no laboratório, das amostras previamente coletadas; c.2) número total de anéis de vasos laticíferos: determinado pelo exame das secções radiais longitudinais.

As variáveis foram analisadas usando-se o modelo estatístico, que considera todos os efeitos aleatórios (exceto a média), consistindo do seguinte:

$\mathrm{Y}_{\mathrm{ijk}}=\mu+\mathrm{p}_{\mathrm{i}}+\mathrm{b}_{\mathrm{j}}+\mathrm{e}_{\mathrm{ij}}+\mathrm{d}_{\mathrm{ijk}}$

onde:

$\mathrm{Y}_{\mathrm{ijk}}$ : observação relativa à planta $\mathrm{k}$, na progênie $\mathrm{i}$, no bloco $\mathrm{k}$, dentro da repetição j;

$\mu$ : média geral;

$\mathrm{p}_{\mathrm{i}}$ : efeito da progênie $\mathrm{i}$;

$b_{j}$ : efeito do bloco $j$;

$\mathrm{e}_{\mathrm{ij}}$ : erro experimental associado à parcela $\mathrm{ij}$; $\mathrm{d}_{\mathrm{ijk}}$ : efeito do indivíduo $\mathrm{k}$ dentro da parcela $\mathrm{ij}$.

As estimativas de parâmetros genéticos e fenotípicos foram obtidas através do software genético-estatístico denominado SELEGEN, desenvolvido por Resende et al. (1994).
Os coeficientes de herdabilidade dos indivíduos na parcela $\left(\mathrm{h}_{d}^{2}\right)$, da média de progênie $\left(\mathrm{h}_{f}^{2}\right)$, da média de parcela $\left(\mathrm{h}_{p}^{2}\right)$, dos indivíduos no bloco $\left(\mathrm{h}_{i b}^{2}\right)$ e de plantas individuais $\left(\mathrm{h}_{i e}^{2}\right)$, associados aos diferentes efeitos do modelo linear, foram estimados pelas seguintes expressões (Resende \& Higa, 1994):

$$
\begin{aligned}
& \left(\mathrm{h}_{d}^{2}\right)=\frac{3 / 4 \dot{\mathrm{o}}_{A}^{2}}{\dot{\mathrm{o}}_{d}^{2}} \\
& \left(\mathrm{~h}_{f}^{2}\right)=\frac{[(3+n b) /(4 n b)] \mathrm{\sigma}_{A}^{2}}{\sigma_{p}^{2}+\frac{\sigma_{e}^{2}}{b}+\frac{\sigma_{d}^{2}}{n b}} \\
& \left(\mathrm{~h}_{p}^{2}\right)=\frac{[3 /(4 n)] \mathrm{\sigma}_{A}^{2}}{\sigma_{e}^{2}+\frac{\sigma_{d}^{2}}{n}} \\
& \left(\mathrm{~h}_{i b}^{2}\right)=\frac{\sigma_{A}^{2}}{\sigma_{p}^{2}+\sigma_{e}^{2}+\sigma_{d}^{2}} \\
& \left(\mathrm{~h}_{i e}^{2}\right)=\frac{\sigma_{A}^{2}}{\sigma_{p}^{2}+\sigma_{e}^{2}+\sigma_{d}^{2}+\sigma_{b}^{2}}
\end{aligned}
$$

onde:

$\sigma_{A}^{2}=4 \sigma_{p}^{2}$

$\sigma_{A}^{2}=$ variância genética aditiva;

$\sigma_{e}^{2}=$ variância ambiental entre parcelas;

$\sigma_{d}^{2}=$ variância fenotípica entre plantas dentro de parcelas;

$\sigma_{p}^{2}=$ variância genética entre progênies;

$n=$ número de plantas/parcela;

$b=$ número de blocos.

O termo coeficiente de predição genética (C.P.G.) equivale a uma herdabilidade indireta para o diferencial de seleção do caráter objetivo da seleção (Resende et al., 1994), envolvendo todos os caracteres. Os coeficientes foram estimados, na análise multivariada, pela seguinte expressão (Resende et al., 1995):

C.P.G. $=r_{(I, A)} \cdot h_{o}$ onde:

$\mathrm{r}_{(\mathrm{I}, \mathrm{A})}=$ acurácia seletiva;

$\mathrm{h}_{\mathrm{o}}=$ raiz quadrada da herdabilidade associada ao caráter objetivo da seleção.

Os coeficientes de variação genética $\left(\mathrm{Cv}_{\mathrm{g}} \%\right)$ e experimental $\left(\mathrm{Cv}_{\mathrm{e}} \%\right)$ foram estimados seguindo-se as fórmulas apresentadas por Vencovsky (1987):

$$
\begin{aligned}
& \mathrm{CV}_{\mathrm{g}}(\%)=\frac{\sqrt{\sigma_{p}^{2}}}{\bar{X}} \cdot 100 \\
& \mathrm{CV}_{\mathrm{e}}(\%)=\frac{\sqrt{\mathrm{o}_{e}^{2}+\mathrm{o}_{d}^{2}}}{\bar{X}} .100 .
\end{aligned}
$$


A estimação de valores genéticos e do progresso genético foram obtidos por meio da seleção individual $\left(\mathrm{I}_{\mathrm{i}}\right)$, entre $\left(I_{e}\right)$, dentro $\left(I_{d}\right)$ e combinada $\left(I_{c}\right)$, sendo utilizadas as expressões apresentadas por Resende \& Higa (1994) como:

$\mathrm{I}_{\mathrm{i}}=\mathrm{h}_{i e}^{2}\left(X_{i j k}-\bar{X}\right)$

$\mathrm{I}_{\mathrm{e}}=\mathrm{h}_{f}^{2}\left(\bar{X}_{i . .}-\bar{X}\right)$

$\mathrm{I}_{\mathrm{d}}=\mathrm{h}_{d}^{2}\left(\mathrm{y}_{\mathrm{ijk}}-\overline{\mathrm{y}}_{\mathrm{ij}}\right)$

$\mathrm{I}_{\mathrm{c}}=h_{d}^{2} \cdot X_{i j k}-h_{d}^{2} \cdot \bar{X}_{i j .}+h_{f}^{2} \cdot \bar{X}_{i . .}-h_{f}^{2} \cdot \bar{X} \ldots$ onde,

$\mathrm{h}_{i e}^{2}$ é o coeficiente de herdabilidade, no sentido restrito, dos indivíduos no experimento; $X_{i j k}$ é o valor do indivíduo k na parcela ij; $\bar{X}$ é a média geral do experimento; $X_{i . .}$ é a média da família; $\bar{X}_{i j . .}$ é a média da parcela; $X_{i j k}$ é o desvio do valor individual (parcela).

O progresso com seleção entre e dentro equivale à somatória dos ganhos obtidos na seleção entre e seleção dentro de progênies.

O progresso com seleção combinada equivale à média dos valores genéticos (índice) dos indivíduos selecionados.

Os estimadores de acurácia derivados por Resende et al. (1995) no que tange aos diferentes métodos de seleção, utilizados no presente trabalho, são os seguintes:

a) Individual: $\left(\frac{\sigma_{A}^{2}}{\sigma_{F_{i e}}^{2}}\right)^{1 / 2}$

b) Entre e dentro (seleção em dois estágios):

b.1. Seleção entre: $\left(\frac{p-1}{p}\right)^{1 / 2} \frac{1+(n b-1) r}{n b} \sigma_{A} / \sigma_{\bar{F}}$

b.2. Seleção dentro: $\left(\frac{n-1}{n}\right)^{1 / 2}(1-r) \sigma_{A} / \sigma_{d}$

c) Combinada: $\left[\frac{n-1}{n}(1-r)^{2} \frac{\sigma_{A}^{2}}{\sigma_{d}^{2}}+\frac{p-1}{p}\left(\frac{1+(n b-1)}{n b}\right)^{2} \frac{\sigma_{A}^{2}}{\sigma_{F}^{2}}\right]^{1 / 2}$

onde:

$\mathrm{r}=$ coeficiente de correlação genética entre indivíduos de uma mesma progênie (1/4 para meios-irmãos);

$\mathrm{n}=$ número de plantas por parcela;

$\mathrm{p}=$ número de progênies.

O parâmetro denominado acurácia é uma medida muito útil de precisão da predição de valores genéticos, e equiva- le à correlação entre os valores genéticos, verdadeiros e os valores genéticos preditos.

\section{RESULTADOS E DISCUSSÃO}

Os resultados obtidos na análise de variância relativos aos caracteres estudados são apresentados na Tabela 1. A análise revelou haver diferenças significativas entre famílias quanto aos caracteres produção de borracha seca (PB), espessura de casca (EC) e circunferência do caule (CC) pelo teste F, a 1\% de probabilidade. Esta variabilidade demonstrada é condição essencial para o estabelecimento de um programa de melhoramento genético, e pode ser efetivamente explorada com vistas ao aumento da produção de borracha. $\mathrm{O}$ caráter número de anéis de vasos laticíferos não manifestou variabilidade significativa a $5 \%$ de probabilidade.

Os coeficientes de variação experimental $\left(\mathrm{CV}_{\mathrm{e}} \%\right)$ obtidos quanto aos caracteres NA $(9,25 \%)$, EC $(14,61 \%)$ e CC $(13,19 \%)$ podem ser considerados baixos e demonstram boa precisão para o ensaio e para critério de avaliação. $\mathrm{OCV}_{\mathrm{e}}$ referente ao caráter PB $(33,45 \%)$ indica que a coleta desses dados está sujeita a erros experimentais de controle relativamente difícil, e expressa propriedades

TABELA 1. Médias e quadrados médios obtidos nas análises de variância dos caracteres número de anéis de vasos laticíferos (NA), produção de borracha seca (PB), espessura de casca (EC) e circunferência do caule (CC), de progênies de $H$. brasiliensis, aos três anos de idade, em Votuporanga, SP.

\begin{tabular}{|c|c|c|c|c|}
\hline \multirow{2}{*}{$\begin{array}{l}\text { Fontes de } \\
\text { variação }\end{array}$} & \multicolumn{4}{|c|}{ Quadrados Médios } \\
\hline & NA & $\mathrm{PB}(\mathrm{g})$ & $\mathrm{EC}(\mathrm{mm})$ & $\mathrm{CC}(\mathrm{cm})$ \\
\hline Blocos & 0,0278 & 0,0424 & 0,2410 & 1,8251 \\
\hline Progênies & $0,0403^{\text {ns }}$ & $0,1162^{* *}$ & $1,3021^{* *}$ & $55,9117^{* *}$ \\
\hline Resíduo & 0,0245 & 0,0281 & 0,2364 & 8,4760 \\
\hline Dentro & 0,2088 & 0,2718 & 2,2158 & 113,7422 \\
\hline Médias & 1,6914 & 0,5013 & 3,3265 & 22,0616 \\
\hline $\mathrm{CV}_{\mathrm{e}}(\%)$ & 9,2495 & 33,4483 & 14,6150 & 13,1965 \\
\hline
\end{tabular}

1 Os graus de liberdade de blocos, progênies e resíduo são, respectivamente, 4,21 e 84 .

${ }^{* *} \mathrm{p}<0,01$.

ns $\mathrm{e}^{* *}$ Não-significativo e significativo a $1 \%$ de probabilidade, respectivamente. 
intrínsecas do caráter. Porém, o referido valor é considerado médio quando comparado às estimativas de Paiva et al. (1982) e Alves et al. (1987), que obtiveram valores da ordem de $38,30 \%$ e $50,44 \%$, respectivamente, e de magnitude condizente à que foi apresentada por Moreti et al. (1994).

O coeficiente de variação genética, que expressa em porcentagem da média geral a quantidade de variação genética existente, apresentou valores mais expressivos quanto ao caráter $\mathrm{PB}$ em relação aos demais. Esses resultados são condizentes com os encontrados por Gonçalves et al. (1983), Moreti et al. (1994) e Boock et al. (1995) no tocante à espécie, o que confirma a variabilidade demonstrada no teste $\mathrm{F}$ relativamente a progênies.

Os valores de herdabilidade (método univariado) e de predição genética (método multivariado), da progênie e da parcela, como também dos indivíduos na parcela, no bloco e no experimento, são apresentados na Tabela 2.

Os valores encontrados variaram entre os caracteres em estudo. Observam-se, nos dois métodos, valores mais elevados de herdabilidade $\mathrm{e}$ de coeficiente de predição genética das progênies quanto aos caracteres PB $(0,803)$, EC $(0,867)$ e CC $(0,899)$.
As estimativas dos coeficientes de herdabilidade e de predição genética dos indivíduos foram menores que as das médias de famílias no que tange a todas as características, o que indica que a seleção pode ser mais efetiva nas médias de progênies do que dentro de progênies. Tais resultados são coerentes com os observados na literatura relativa a outras espécies florestais (Matzires \& Zobel, 1973; Kageyama, 1980; Sturion, 1993; Sampaio, 1996) e quanto à seringueira (Gilbert et al., 1973; Nga \& Subramanian, 1974; Moreti et al., 1994; Boock et al., 1995).

Os valores de herdabilidade, obtidos nas progênies sugerem grandes possibilidades de ganho genético, tendo em vista que o progresso esperado pela seleção depende da herdabilidade do caráter, da intensidade de seleção, e, de forma inversa, do desvio fenotípico do caráter (Dudley \& Moll, 1969).

Os coeficientes de predição genética (método multivariado) foram superiores aos coeficientes de herdabilidade, o que evidencia a importância da utilização de informações referentes a outros caracteres, correlacionados com a expressão do caráter desejado. Para a seringueira, torna-se uma situação interessante, tendo em vista que o objetivo principal dos programas de melhoramento da espécie é a

TABELA 2. Coeficientes de herdabilidade (método univariado) e de predição genética (método multivariado), associados a diferentes efeitos empregados nos métodos de seleção para os quatro caracteres de $H$. brasiliensis, aos três anos de idade em Votuporanga, $\mathrm{SP}^{1}$.

\begin{tabular}{|c|c|c|c|c|c|c|c|c|}
\hline \multirow[t]{2}{*}{ Efeitos } & \multicolumn{4}{|c|}{ Herdabilidade } & \multicolumn{4}{|c|}{ Coeficientes de predição genética } \\
\hline & NA & PB & $\mathrm{EC}$ & $\mathrm{CC}$ & NA & PB & $\mathrm{EC}$ & $\mathrm{CC}$ \\
\hline Indivíduos na parc. $\left(h_{i p}^{2}\right)$ & 0,045 & 0,194 & 0,288 & 0,250 & 0,134 & 0,214 & 0,311 & 0,281 \\
\hline Progênies $\left(h_{f}^{2}\right)$ & 0,417 & 0,803 & 0,867 & 0,889 & 0,653 & 0,806 & 0,872 & 0,902 \\
\hline Parcela $\left(h_{p}^{2}\right)$ & 0,039 & 0,188 & 0,270 & 0,336 & 0,125 & 0,202 & 0,296 & 0,348 \\
\hline Indivíduos no bloco $\left(h_{i b}^{2}\right)$ & 0,059 & 0,243 & 0,349 & 0,315 & 0,166 & 0,263 & 0,372 & 0,346 \\
\hline Indivíduos no exp. $\left(h_{i e}^{2}\right)$ & 0,059 & 0,242 & 0,349 & 0,316 & 0,166 & 0,263 & 0,372 & 0,346 \\
\hline
\end{tabular}

${ }^{1}$ NA: número de anéis de vasos laticíferos; PB: produção de borracha seca; EC: espessura de casca; CC: circunferência do caule. 
obtenção de material genético com alto potencial de produção.

As acurácias, ganhos genéticos diretos e nova média da população, associados ao método univariado dos caracteres em estudo, são apresentados na Tabela 3 .

Os ganhos genéticos estimados na seleção entre e dentro de progênies foram: NA $=3,57 \%$; $\mathrm{PB}=70,197 \%$; $\mathrm{EC}=30,684$ e $\mathrm{CC}=28,368 \%$, elevando a nova média da população para o caráter $\mathrm{PB}$, após um ciclo de seleção, de $0,50 \mathrm{~g}$ para $0,68 \mathrm{~g}$.

Os ganhos genéticos estimados na seleção individual foram: $\mathrm{NA}=2,42 \% ; \mathrm{PB}=94,74 \% ; \mathrm{EC}=31,61 \%$; $\mathrm{CC}=27,29 \%$, elevando a nova média da população do carácter PB, após um ciclo de seleção, de $0,50 \mathrm{~g}$ para $0,97 \mathrm{~g}$.

A seleção combinada proporcionou ganhos genéticos de: $\mathrm{NA}=3,72 \%, \mathrm{~PB}=95,94 \% ; \mathrm{EC}=37,76 \%$; $\mathrm{CC}=33,19 \%$. Os ganhos estimados elevaram a nova média da população para o caráter $\mathrm{PB}$, após um ciclo de seleção, de $0,50 \mathrm{~g}$ para $0,98 \mathrm{~g}$.

As estimativas obtidas no caráter PB apresentaram os valores mais expressivos de ganhos genéticos entre os caracteres estudados, o que denota o grande potencial da população em termos de produção de borracha e sugere grandes perspectivas para o programa de melhoramento da seringueira.

Comparando-se as acurácias e os ganhos genéticos estimados pela seleção entre e dentro, individual, e seleção combinada, constata-se que a seleção combinada proporcionou ligeira superioridade para todos os caracteres. Esses resultados estão inseridos no contexto das recomendações de Cotterill \& Dean (1990), de que a seleção combinada é mais adequada para os casos em que a herdabilidade dos indivíduos é baixa, propiciando ganhos mais elevados com a seleção. Neste trabalho, as estimativas de herdabilidade dos indivíduos, no experimento, quanto aos caracteres $\mathrm{PB}, \mathrm{EC}$ e CC, são consideradas médias, segundo classificação de Resende (1997), o que explicaria os ganhos adicionais de pequena magnitude. A superioridade demonstrada pela seleção combinada é coerente com os resultados obtidos no tocante a outras espécies florestais por Bueno Filho (1992), Resende \& Araujo (1993), Resende \& Bertolucci (1993), Sturion (1993), Sturion et al. (1994), Cornacchia et al. (1995), Pires et al. (1996) e Sampaio (1996).

Os ganhos adicionais obtidos pela seleção combinada foram, percentualmente, de pequena magnitude, em relação à seleção individual. Porém, a seleção combinada deve ser utilizada por propiciar maior acurácia e ganho genético, e não implicar custos adicionais ao processo seletivo. Além disso, pequenos ganhos podem ser significativos, considerando-se que os plantios da seringueira ocupam grandes áreas.

As acurácias, ganhos genéticos e nova média da população associados ao método multivariado das características em estudo, são apresentados na Tabela 4.

Os ganhos genéticos estimados na seleção entre e dentro de progênies foram: $\mathrm{NA}=8,85 \%$; $\mathrm{PB}=71,139 \% ; \mathrm{EC}=33,210$ e $\mathrm{CC}=32,584 \%$, elevando a nova média da população do caráter $\mathrm{PB}$, após um ciclo de seleção, de $0,50 \mathrm{~g}$ para $0,79 \mathrm{~g}$.

Os ganhos genéticos estimados na seleção individual foram: $\mathrm{NA}=9,46 \% ; \mathrm{PB}=96,29 \% ; \mathrm{EC}=34,17 \%$; $\mathrm{CC}=33,03 \%$. O ganho estimado elevou a nova média da população para o caráter $\mathrm{PB}$, após um ciclo de seleção, de $0,50 \mathrm{~g}$ para $0,98 \mathrm{~g}$.

A seleção combinada proporcionou ganhos genéticos de: $\mathrm{NA}=9,95 \% ; \mathrm{PB}=98,95 \%$; $\mathrm{EC}=38,78 \%$; $\mathrm{CC}=37,20 \%$. O ganho estimado elevou a nova média da população para o caráter $\mathrm{PB}$, após um ciclo de seleção, de $0,50 \mathrm{~g}$ para $0,99 \mathrm{~g}$.

De forma similar aos resultados obtidos pelo método univariado, as acurácias e ganhos genéticos estimados pela seleção combinada, no método

TABELA 3. Acurácias e ganhos genéticos diretos (\%), associados aos métodos de seleção univariados dos quatro caracteres de $\boldsymbol{H}$. brasiliensis, aos três anos de idade, em Votuporanga, SP.

\begin{tabular}{|c|c|c|c|c|c|c|c|}
\hline \multirow[t]{3}{*}{ Caracteres $^{1}$} & \multicolumn{3}{|c|}{ Entre e dentro } & \multicolumn{2}{|c|}{ Individual } & \multicolumn{2}{|c|}{ Combinada } \\
\hline & \multicolumn{2}{|c|}{ Acurácia } & \multirow{2}{*}{$\begin{array}{c}\text { Ganho } \\
(\%)\end{array}$} & \multirow[t]{2}{*}{ Acurácia } & \multirow{2}{*}{$\begin{array}{c}\text { Ganho } \\
(\%)\end{array}$} & \multirow[t]{2}{*}{ Acurácia } & \multirow{2}{*}{$\begin{array}{c}\text { Ganho } \\
(\%)\end{array}$} \\
\hline & Entre & Dentro & & & & & \\
\hline NA & 0,325 & 0,175 & 3,567 & 0,242 & 2,424 & 0,369 & 3,725 \\
\hline $\mathrm{PB}$ & 0,451 & 0,362 & 70,197 & 0,492 & 94,743 & 0,578 & 95,940 \\
\hline $\mathrm{EC}$ & 0,468 & 0,441 & 30,684 & 0,591 & 31,614 & 0,644 & 37,757 \\
\hline $\mathrm{CC}$ & 0,477 & 0,411 & 28,368 & 0,562 & 29,290 & 0,629 & 33,192 \\
\hline
\end{tabular}


TABELA 4. Acurácias e ganhos genéticos diretos (\%), associados aos métodos de seleção multivariados dos quatro caracteres de $\boldsymbol{H}$. brasiliensis, aos três anos de idade, em Votuporanga, SP.

\begin{tabular}{|c|c|c|c|c|c|c|c|}
\hline \multirow[t]{3}{*}{ Caracteres $^{1}$} & \multicolumn{3}{|c|}{ Entre e dentro } & \multicolumn{2}{|c|}{ Individual } & \multicolumn{2}{|c|}{ Combinada } \\
\hline & \multicolumn{2}{|c|}{ Acurácia } & \multirow{2}{*}{$\begin{array}{c}\text { Ganho } \\
(\%)\end{array}$} & \multirow[t]{2}{*}{ Acurácia } & \multirow{2}{*}{$\begin{array}{c}\text { Ganho } \\
(\%)\end{array}$} & \multirow[t]{2}{*}{ Acurácia } & \multirow{2}{*}{$\begin{array}{c}\text { Ganho } \\
(\%)\end{array}$} \\
\hline & Entre & Dentro & & & & & \\
\hline NA & 0,509 & 0,515 & 8,849 & 0,684 & 9,460 & 0,724 & 9,952 \\
\hline PB & 0,452 & 0,399 & 71,139 & 0,535 & 96,289 & 0,613 & 98,953 \\
\hline $\mathrm{EC}$ & 0,471 & 0,475 & 33,210 & 0,630 & 34,169 & 0,669 & 38,779 \\
\hline $\mathrm{CC}$ & 0,478 & 0,461 & 32,584 & 0,684 & 33,032 & 0,724 & 37,196 \\
\hline
\end{tabular}

1 NA: número de anéis de vasos laticíferos; PB: produção de borracha seca; EC: espessura de casca; CC: circunferência do caule.

multivariado, foram levemente superiores aos estimados pela seleção individual.

Comparando-se os valores encontrados pelo método de seleção univariado (Tabela 3) e multivariado (Tabela 4), constata-se que os valores estimados de acurácias e ganhos genéticos pelo método multivariado mostraram-se superiores. Diferença bem expressiva foi encontrada quanto ao caráter NA, o que evidencia que outros caracteres considerados nos cálculos do método multivariado têm valores de herdabilidade maiores, e, provavelmente, alta correlação com o caráter NA. Tais resultados reafirmam a importância da utilização de informações referentes a outros caracteres, aumentando a acurácia na avaliação do caráter desejado.

\section{CONCLUSÕES}

1. Os coeficientes de predição genética superiores aos coeficientes de herdabilidade evidenciam a importância da utilização de informações referentes a outros caracteres correlacionados com a expressão do caráter desejado.

2. O método de seleção combinada é promissor e deve ser utilizado, pois propicia maior acurácia e ganho genético.

\section{REFERÊNCIAS}

ALVES, M.R.; BANDEL, G.; VENCOVSKY, R. Avaliação de seleção em seringueira (Hevea spp.). Boletim da Faculdade de Ciências Agrárias do Pará, Belém, v.16, p.53-63, 1987.

BOOCK, M.V.; GONÇALVES, P. de S.; BORTOLETTO, N.; MARTINS, A.L.M. Herdabilidade, variabilidade genética e ganhos genéticos para produção e caracteres morfológicos em progênies jovens de seringueira. Pesquisa Agropecuária Brasileira, Brasília, v.30, n.5, p.673-681, 1995.
BUENO FILHO, J.S.S. Seleção combinada versus seleção seqüencial no melhoramento de populações florestais. Piracicaba : ESALQ-USP, 1992. 96p. Dissertação de Mestrado.

CORNACCHIA, G.; CRUZ, C.D.; PIRES, W. Seleção combinada e seleção entre e dentro de famílias de meios-irmãos de três espécies do gênero Pinus. Revista Árvore, Viçosa, v.19, n.2, p.200-212, 1995.

COTTERILL, P.P.; DEAN, C.A. Successful tree breeding with index selection. Sidney : CSIRO, Division of Forest and Forest Products, 1990. 80p.

DUDLEY, J.W.; MOLL, R.H. Interpretation and use of estimation of heritability and genetic variance in plant breeding. Crop Science, Madison v.2, n.3, p.257$262,1969$.

FALCONER, D.S. Introdução à genética quantitativa. Viçosa : Universidade Federal de Viçosa, 1987. 279p.

GILBERT, N.E.; DODDS, K.S.; SUBRAMANIAN, S. Progress of breeding investigations with Hevea brasiliensis. Analysis of data from earlier crosses. Journal of the Rubber Research Institute of Malaysia, Kuala Lumpur, v.3, n.5, p.365-380, 1973.

GONÇALVES, P. de S.; CARDOSO, M.; COLOMBO, C.A.; ORTOLANI, A.A.; MARTINS, A.L.M.; SANTOS, I.C.I. dos. Variabilidade genética da produção anual da seringueira: estimativas de parâmetros genéticos e estudo de interação genótipo $\mathrm{x}$ ambiente. Bragantia, Campinas, v.49, n.2, p.305-320, 1990.

GONÇALVES, P. de S.; ROSSETTI, A.G.; VALOIS, A.C.C.; VIEGAS, I. de J.M. Coeficiente de determinação genotípica e estimação de outros parâmetros em clones de seringueira. Pesquisa Agropecuária Brasileira, Brasília, v.18, n.5, p.527-532, 1983.

KAGEYAMA, P.Y. Variação genética em progênies de uma população de Eucalyptus grandis (Hill) Maiden. Piracicaba : ESALQ-USP, 1980. 125p. Tese de Doutorado. 
LUSH, J.L. Animal breeding plans. 3.ed. Ames : State College, 1945. 443p.

LUSH, J.L. Family merit and individual merit as bases for selection. American Naturalist, v.81, p.241-261, 1947.

MATZIRES, D.I.; ZOBEL, B.J. Inheritance and correlations of juvenile characteristics in loblolly Pine. Silvae Genetica, Frankfurt, v.21, p.44-48, 1973.

MORETI, D.; GONÇALVES, P. de S.; GORGULHO, E.P.; MARTINS, A.L.M.; BORTOLETTO, N. Estimativas de parâmetros genéticos e ganhos esperados com a seleção de caracteres juvenis em progênies de seringueira. Pesquisa Agropecuária Brasileira, Brasília, v.29, n.7, p.1099-1109, 1994.

NGA, B.H.; SUBRAMANIAN, S. Variation in Hevea brasiliensis. I. Yield and girth data of the 1937 hand pollinated seedlings. Journal of the Rubber Research Institute of Malaysia, Kuala Lumpur, v. 24, n.2, p.69-74, 1974.

PAIVA, J.R. Variabilidade enzimática em populações naturais de seringueira. Piracicaba : ESALQ-USP, 1992. 145p. Tese de Doutorado.

PAIVA, J.R.; KALIL FILHO, A.N. Melhoramento genético da seringueira. Informe Agropecuário, Belo Horizonte, v.11, n.121, p.52-57, 1985.

PAIVA, J.R.; MIRANDA FILHO, J.B.; SIQUEIRA, E.R.; VALOIS, A.C.C. Predição do ganho de alguns caracteres em seringueira em três esquemas de seleção. Pesquisa Agropecuária Brasileira, Brasília, v.17, n.11, p.1646-1653, 1982.

PEREIRA, J.P. Seringueira: formação de mudas, manejo e perspectivas no noroeste do Paraná. Londrina : IAPAR, 1992. 60p. (IAPAR. Circular técnica, 70).

PIRES, I.E.; CRUZ, C.D.; BORGES, R.C.G.; REGAZZI, A.J. Índice de seleção combinada aplicado ao melhoramento genético de Eucalyptus spp. Revista Árvore, Viçosa, v.20, n.2, p.191-197, 1996.

RESENDE, G.D.S.P.; BERTOLUCCI, F.L.G. Uso da seleção combinada na determinação da eficiência da seleção precoce em progênies de meio-irmãos de Eucalyptus urophylla. In: CONGRESSO FLORESTAL PANAMERICANO, 1., 1993, Curitiba. Anais. Curitiba : SBS-SBEF, 1993. v.1, p.158-160.

RESENDE, M.D.V. Avanços da genética biométrica Florestal. In: ENCONTRO SOBRE TEMAS DE GENÉTICA E MELHORAMENTO, 14., 1997, Piracicaba. Anais. Piracicaba : ESALQ-USP, 1997. p.150-158.

RESENDE, M.D.V.; ARAUJO, A.J. Modelo genéticoestatístico e delineamento experimental para estimação de componentes da variação genética e parâmetros genéticos em teste de progênies com indivíduos repetidos clonalmente. Revista Floresta, Curitiba, v.23, n.1/2, p.35-45, 1993.

RESENDE, M.D.V.; ARAUJO, A.J.; SAMPAIO, P.T.B.; WIECHETECK, A.S. Acurácia seletiva, intervalos de confiança e variância de ganhos genéticos associados a 22 métodos de seleção em Pinus caribaea var. hondurensis. Revista Floresta, Curitiba, v.24, n.1/2, p.35-45, 1995.

RESENDE, M.D.V.; HIGA, A.R. Maximização da eficiência da seleção em testes de progênies de Eucalyptus através da utilização de todos os efeitos do modelo matemático. Boletim de Pesquisa Florestal, Curitiba, n.28/29, p.37-56, 1994

RESENDE, M.D.V.; OLIVEIRA, E.B.; MELINSKI, L.C.; GOULART JUNIOR, F.S.; OAIDA, G.R.P. Seleção genética computadorizada - Selegen "Best Prediction": manual do usuário. Colombo : Embrapa-CNPF, 1994. 31p.

SAMPAIO, P.T.B. Variação genética entre procedências e progênies de Pinus oocarpa Schiede, Pinus caribaea var. hondurensis Barr. \& Golf. e Pinus maximinoi, H.E. Moore e métodos de seleção para melhoramento genético. Curitiba : SEA/UFPR, 1996. 169p. Tese de Doutorado.

SOCIEDADE BRASILEIRA DE SILVICULTURA. A realidade da seringueira no Brasil. Silvicultura, v.15, n.61, p.27-29, 1995.

STURION, J.A. Variação genética de características de crescimento e de qualidade da madeira em progênies de Eucalyptus viminalis. Curitiba : UFPR, 1993. 112p. Tese de Doutorado.

STURION, J.A.; RESENDE, M.D.V.; CARPANEZZI, A.A.; ZANON, A. Variação genética e seleção para características de crescimento em teste de progênies de Mimosa scabrella var. aspericarpa. Boletim de Pesquisa Florestal, n.28/29, p.73-83, 1994.

TAN, H.; SUBRAMANIAN, S. A five-parent diallel cross analysis for certain characters of young Hevea seedlings. In: INTERNATIONAL RUBBER CONFERENCE, 1975, Kuala Lumpur. Proceedings. Kuala Lumpur : RRIM, 1976. v.2, p.13-16.

VENCOVSKY, R. Herança quantitativa. In: PATERNIANI, E.; VIEGAS, G.P. (Eds.). Melhoramento e produção do milho. 2.ed. Campinas : Fundação Cargill, 1987. v.1, cap.5, p.137-214.

VIRGENS FILHO, A.C. Seringueira-Programa Regional de Pesquisa. Ilhéus : CEPEC/CEPLAC, 1990. 32p. 